\title{
Personalia
}

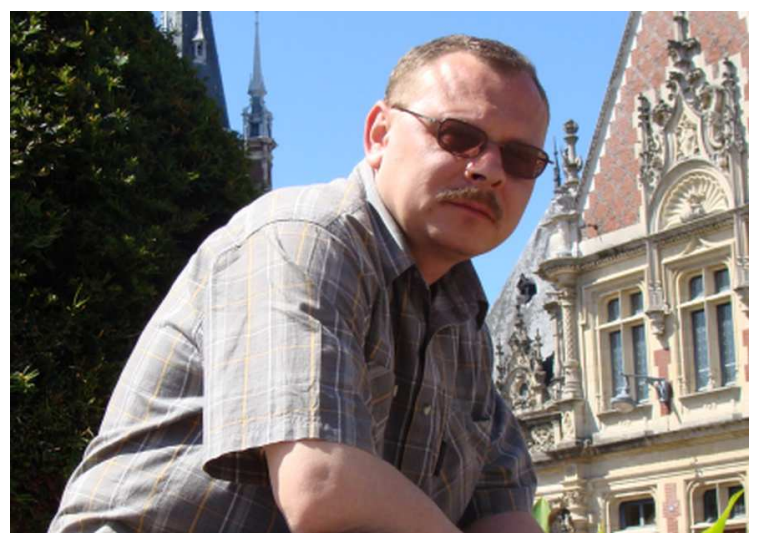

\section{In memory of Eduard Vakarin}

On May 22, 2015 a sudden regrettable death took away Eduard Vakarin at an early age. Although Vakarin never held a prominent academic position, he established a reputation in the statistical mechanical theory of Condensed Matter by his investigation of different real coupled systems. The results he obtained are significant from both fundamental and practical perspectives.

E. Vakarin was born in Ukraine in the city of Lviv on February 28, 1967. After finishing the Lviv University in 1992, he started his scientific activities under the supervision of M. Holovko as a postgraduate student at the Institute of Condensed Matter Physics (ICMP) of the National Academy of Sciences of Ukraine (NASU). In 1996 he defended his Ph. D. dissertation entitled "The influence of association phenomena on the interfacial properties”. Later on, from 1996 till 2001, he worked as a researcher at the ICMP of NASU. From 2001 till 2015 he continued his scientific activities as the first class researcher of CNRS in the group of J.P. Badiali at the Université Pierre et Marie Curie and the École Nationale Supérieure de Chimie de Paris in Paris, France. Here in 2008 he defended his habilitation thesis entitled "Theoretical approaches for the description of real systems. Statistical mechanics of coupled systems".

In early works by E. Vakarin, for the description of associative fluids near a surface, the associative version of the Henderson-Abraham-Barker approach was developed. Within the framework of this approach, the drying effect of a fluid from the surface due to the association of the fluid was found. For a crystalline surface, due to the competition between intramolecular correlation and attraction between molecules with lattice sites, there was found the possibility to have cooperative adsorption of fluid at surprisingly rather small densities. This phenomenon was used to explain the anomalous adsorption of polymers in the diluted-semidiluted concentration regime and to clarify the mechanism of low-pressure insertion and separation of chain-line molecules in host matrices. E. Vakarin together with Yu. Duda reformulated the associative integral equation theory for network forming fluids. The liquid-gas phase transition in such fluids was found and it was shown that the liquid phase including the critical point is entirely embedded in the percolation region. It is also shown that the crystalline surface enhances the clustering in fluids. For the non-rigid surface it was possible to investigate the modification of the surface by absorbent. In this study it was shown that the presence of an absorbent may induce distortive and roughening transitions on the underlying substrate, a structural rearrangement of surface and some other complex phenomena due to the competing adsorbent-substrate interactions. Within the framework of this research, the effect of ice films morphology on $\mathrm{HCl}$ uptake was investigated as a possible mechanism of chlorine accumulation in polar stratospheric clouds.

Another important aspect of E. Vakarin's activities is connected with the investigations of the physics associated with intercalation of ions into host matrixes. He extensively used the fact that an insertion pro- 
cess can be considered as 3D adsorption. In particular, for the first time he explicitly took into account the fact that a change on the volume of the host material may have a strong effect on the insertion mechanism. Employing the lattice gas model, combined with the linear elasticity theory, Eduard argues that the major feature of the intercalation isotherms can be well understood in terms of a coupling between the configurational and structural transitions. The theory developed provides a quantitative description of different insertion processes and was used for the interpretation of the experiments obtained in the teams of M.D. Levi (Bar-Ilan University, Israel) and G. Garcia-Belmonte (Universitat Jaume 1, Spain). The idea regarding the deformation of a matrix by adsorption of fluid was used in collaboration with W. Dong (ENS Lyon, France) for investigation of some aspects concerning phase transition in porous materials. The question was related to the following: how should we characterise a porous material simply beyond the porosity and why computer simulations can give divergent results.

From this kind of study Eduard decided to investigate fundamental problems related to the entropy in complex systems and in non-extensive media. Statistical properties of such systems were studied within a combination of the maximum information principle and the super-statistical approach with parametrized information entropy measures such as Tsallis or Renyi. In his latest work, E. Vakarin developed the theoretical tools needed to investigate the process of aging in lithium batteries. This is not only a very important subject for producers of batteries but also a very fundamental problem to be solved.

We present our condolences to the family of E. Vakarin, to colleagues and friends in connection with his untimely death.

J.P. Badiali, D. di Caprio, M.F. Holovko 